\title{
LÂMINAS DE ÁGUA E DOSES DE NITROGÊNIO NA PRODUÇÃo DE AVEIA (Avena sativa $L$.) PARA FORRAGEM
}

\author{
J. A. FRIzZONE', R. E. F. TEODORO ${ }^{2}$, A. S. PEREIRA ${ }^{3}$ e T. A. BOTREL' \\ 'Dep. de Engenharia. Rural - ESALQ/USP, C.P. 9, CEP:13418-900, Piracicaba SP \\ ${ }^{2}$ Dep. de Agnonomia - UFU, CEP: 38.40a-902, Uberiandia, MG \\ ${ }^{3}$ Pós graduando em Irrigafáo e Drenagem - ESALQ/USP, Ptracicaba, SP
}

RESUMO - Foi realizado experimento visando estudar os efeitos da irrigaç̆o suplementar e da adubaç.o nitrogenada na produtividade de aveia (Avena sattva L.), variedàde UPF7, para forragem. 0 delineamento experimental foi o de blocos ao acaso com parcelas subdivididas, com quatro repetif̧óes. Os tratamentos constaram da combinação de quatro làminas de irrigação aplicadas quando a eraporação acumulada no tanque "Classe $A$ " (ECA) atingia aproximadamente $30 \mathrm{~mm}(I=0 \%, I=50 \%, I=100 \%$ e I = 150\% de BCA) e quatro níveis de adubaçăo nitrogenada ( $N=0, N=80, N=160$ e $N=240 \mathrm{~kg}^{-h^{-1}}$ de nitrogenio). A máxima produtividade total de matéria seca (6,9 tha:-1) foi estimada para 319,2 mm de agua e 152,4 kg.ha-1 de nitrogenio. Aos preces considerados para insumos e produto, a maxima receita líquida ( $R \$ 897 . \mathrm{ha}^{-1}$ ) foi obtida para $310 \mathrm{~mm}$ de agua $120 \mathrm{~kg}^{-h^{-1}}$ de $^{2}$ nitrogenio.

Descritores: irrigação suplementar, adubaçăo nitrogenada, níreis otimos, receita líquida.

\section{IRRIGATION DEPTHS AND NITROGEN LEVELS FOR FORAGE OAT (Avena sativa L.) PRODUCTION}

\begin{abstract}
This study was conducted to determine the effects of supplemental irrigation and nitrogen fertilization on forage oat (Avena sattra, L.) productivity, variety UPF7. The experiment was a split-plot randomized block design with four replications. The experimental unit consisted of combinations of four irrigation depths applied when cumulative Classe A pan evaporation (ECA) approximately reached $30 \mathrm{~mm}, \quad(I=0 \%, I=50 \%, 1=100 \%$ and $I=150 \%$ of ECA), and four nitrogen levels $\left(N=0, N=80, N=160\right.$ e $\left.N=240 \mathrm{~kg} \cdot \mathrm{ha}^{-1}\right)$. The total maximum productivity of iry matter $\left(6,9 \mathrm{t}^{\left.-\mathrm{ha}^{-1}\right)}\right.$ was obtained for the water depth of $319,2 \mathrm{~mm}$ and $152,4 \mathrm{~kg}_{\mathrm{ha}} \mathrm{h}^{-1}$ of nitrogen. For the considered prices, the water and nitrogen levels that induced the maximum net income were $310 \mathrm{~mm}$ and $120 \mathrm{~kg} \cdot \mathrm{ha}^{-1}$, respectively, reaching a maximum net return of R\$ $897 . \mathrm{ha}^{-1}$.

Key words: suplemental irrigation, nitrogen fertilization, optimal levels, net return.
\end{abstract}

\section{INTRODUÇÃo}

A aveia é uma gramínea de inverno, cultivada para produção de grãos e forragem e, ainda, para adubação verde. Os estados do Rio Grande do Sul, Santa Catarina e Paraná são aqueles com maiores áreas cultivadas com aveia, visando às várias finalidades de produção. No Mato Grosso do Sul e Sul de Minas Gerais, o cultivo destina-se basicamente à produção de forragem verde.

As principais espécies cultivadas no País são a aveia branca (Avena sativa L.), a aveia amarela (avena byzantina $C$. Koch) e a aveia preta (Avena strigosa Schreb). As aveias branca e amarela são utilizadas para produção de forragem e grãos, já a aveia preta é indicada para a produção de forragem (FLOSS, 1988).

$A$ aveia pode ser cultivada em quase todos os tipos de solo, com preferência àqueles com altos teores de matéria orgânica, permeáveis e bem drenados. Adapta-se a regiōes tropicais, temperadas e frias, sendo mais indicada para altitudes de $\mathbf{1 0 0 0}$ a $3000 \mathrm{~m}$, podendo-se obter dois ou mais cortes por ano, dependendo da disponibilidade de água no solo (CROWDER et al. ,1967; FLOSS, 1988).

A principal limitação do cultivo de aveia no inverno é a seca. VILELA et al. (1970), consideram que a produção de forragem de aveia, no inverno, só pode ser justificada quando houver condiçōes para irrigação. Conforme pesquisas 
conduzidas na Austrália, a aveia chega a produzir de 4 a 5 t.ha $^{-1}$ de matéria seca, sem irrigação e, quando irrigada, pode alcançar produções que vão além de 11 t.ha- ${ }^{-1}$ (BLOUNT \& FISHER, 1976). No Brasil, as maiores produtividades de matéria seca de aveia, registradas na literatura consultada, são da ordem de 8,0 t.ha- ${ }^{-1}$. Entre tais registros, destaca-se o trabalho de GODOY \& BATISTA (1989), visando recomendar cultivares de aveia forrageira para a região de São Carlos (SP), sob irrigação. Entre a seis cultivares selecionadas para o teste final, em dois cortes, conseguiram produções de matéria seca variando entre 6,828 a 8,802 t.ha $^{-1}$ sendo que a produção do segundo corte foi sempre superior ao do primeiro.

Para avaliar o efeito da irrigação na produção de aveia, LAL (1987) conduziu experimento durante dois anos na Índia. Estabeleceu níveis de irrigação baseados na evaporação do tanque "Classe $A$ " (ECA): 0,$4 ; 0,6 ; 0,8 ; 1,0$ e 1,2 ECA aplicados quando eram acumulados $60 \mathrm{~mm}$ de evaporação do tanque. Concluiu que a produção de matéria seca não foi a fetada pela irrigação e a de matéria verde foi significativamente afetada. No primeiro ano a maior produção foi obtida com o nível de irrigação de 1,0 ECA e no segundo com 1,2 ECA, este não diferindo significativamente de 0,8 e 1,0 ECA.

Visando a produção de forragem com aveia, HUKKERI et al. (1977) trabalharam com três doses de nitrogênio $\left(30,50\right.$, e $\left.90 \mathrm{~kg} \cdot \mathrm{ha}^{-1}\right)$ e três níveis de água, com irrigações efetuadas quando a água disponível no solo, na profundidade de 15 a $30 \mathrm{~cm}$, atingia 25,50 e $75 \%$. Em três anos de estudo concluíram ser positiva a interação entre nitrogênio e água, com produção máxima para o tratamento $75 \%$. Quanto ao nitrogênio, apresentou resposta quadrática com produtividade máxima para 80 kg.ha ${ }^{-1}$.

Para avaliar os efeitos de lâminas de irrigação e doses de nitrogênio na produção da aveia, GILL \& MALIK (1983) conduziram experimento durante dois anos na India. As irrigações foram realizadas com base na evaporação acumulada do tanque "Classe $A$ " $(60,90$ e 120 $\mathrm{mm}$ ) $e$ as doses de nitrogênio foram 0,60 e 120 $\mathrm{kg} \cdot \mathrm{ha}^{-1}$. Encontraram maiores produções de matéria verde e seca para lâmina de irrigação de $60 \mathrm{~mm}$ e dose de nitrogênio de $120 \mathrm{~kg} \mathrm{ha}^{-1}$.

Em Coronel Pacheco-MG, ALVIM et al. (1987) avaliaram o efeito da adubação nitrogenada na produção de matéria seca da aveia
(Avena sativa L.), irrigada por aspersão. As doses de nitrogênio aplicadas foram $0,100,200,300 \mathrm{e}$ $400 \mathrm{~kg} \cdot \mathrm{ha}^{-1}$. Aṕs três cortes, conclaíram que a aplicação de 100 kg.ha ${ }^{-1}$ aumentou significativamente a produção de matéria seca total, registrando produções semelhantes entre esse nivel e os subseqũentes. Tais produções de matéria seca foram $2.983,4.394,4.900$ e $4.871 \mathrm{~kg} \cdot \mathrm{ha}^{-1}$, respectivamente, para os níveis de nitrogênio.

O excesso de água no solo é prejudicial ao desenvolvimento da aveia, pois reduz o perfilhamento e torna as plantas clor 6 fitas, diminuindo a produção de massa verde (LEITE, 1986). A intensidade do dano provocado depende do estádio de desenvolvimento da cultura, da duração do período de excesso, da temperatura e do tipo de solo. CRUCIANI \& MINAMI (1984) estudaram os efeitos do excesso temporário de água no solo sobre a produção de aveia forrageira (Avena strigosa Sckreb), em fases distintas de seu ciclo vegetativo, e o tempo máximo de tolerância da cultura ao excesso de água. Concluíram que apenas no segundo corte o excesso de água afetou significativamente a produção com efeitos proporcionais à duração do excedente e que a aveia não tolera nem um dia de saturação do solo aos 90 dias após a semeadura, mas em qualquer fase dois dias são excessivos.

Os efeitos da adubação nitrogenada $e$ da irrigação, mostram a importância do uso simultâneo dessas duas práticas. Assim, existe grande interesse em estudar o comportamento da aveia, quanto a produção de matéria seca, ao se aplicar diferentes níveis de adubação nitrogenada e de irrigação. Este trabalho tem o objetivo de analisar os efeitos da irrigação suplementar e da adubação nitrogenada na produção de forragem de aveia e gerar informações que permitam a alocação técnica e eficiente desses fatores, na região de Pirassununga, SP.

\section{MATERIAL E METODOS}

O experimento foi conduzido no período de abril a julho de 1989 , no Campo Experimental da Faculdade de Medicina Veterinária e Zootecnia da Universidade de São Paulo, Pirassununga (SP), num Latossolo VermelhoEscuro. A região situa-se a $21^{\circ} 59^{\prime \prime}$ de latitude sul e $47^{\circ} 26^{\prime \prime}$ de longitude oeste. A altitude $\epsilon$ de 634 $m$, apresentando clima temperado chuvoso, com inverno seco e verão quente e chuvoso, tipo Cwa 
pela classificação de Köppen (OLIVEIRA \& PRADO, 1984). Apresenta temperaturas máximas entre 26 e $28^{\circ} \mathrm{C}$ e mínimas entre 16 e $18^{\circ} \mathrm{C}$, com média anual de $22^{\circ} \mathrm{C}$ e precipitação pluviométrica média anual de $1.298 \mathrm{~mm}$ (NASCIMENTO \& PEREIRA, 1988).

Utilizou-se aveia branca (Avena sativa L.) cv. UPF7. O preparo do solo consistiu de duas arações e duas gradagens. A calagem foi realizada com distribuição a lanço de 2 t.ha ${ }^{-1}$ de calcário, visando elevar a saturação de bases a $60 \%$.

A semeadura foi realizada manualmente, nos dias 09 e 10 de abril, no espaçamento de $0,20 \mathrm{~m}$ entre linhas e 80 sementes úteis por metro linear. Realizou-se adubação no sulco de plantio, na base de 80 kg.ha-1 de $\mathrm{P}_{2} \mathrm{O}_{5}$ e 60 kg.ha ${ }^{-1}$ de $K_{2} \mathrm{O}$. A adubação nitrogenada foi feita com sulfato de amônio e as doses de nitrogênio foram preestabelecidas de acordo com os tratamentos, sendo parceladas da seguinte forma: 1/3 na semeadura, 1/3 aos 30 dias após a germinação e 1/3 após o primeiro corte.

$O$ delineamento experimental utilizado foi em blocos casualizados, com parcelas subdivididas. A área útil de cada parcela foi de $2,00 \mathrm{~m}^{2}$, tendo cinco linhas de plantas. Os tratamentos constaram da combinação de quatro níveis de irrigação $\left(I_{1}\right)$ localizados nas parcelas e quatro níveis de nitrogênio $\left(N_{1}\right)$ localizadas nas subparcelas. As irrigações foram aplicadas com base na diferença entre os valores acumulados de evaporação no tanque "Classe $A^{\text {" }}$ (ECA) e os valores de precipitação pluvial (PREC), ocorridos num determinado príodo. Quando essa diferença (I) atingiu aproximadamente $30 \mathrm{~mm}$ foram realizadas irrigações, tendo-se utilizados os seguintes níveis: $b_{6}=0 \%$ de $I, I_{1}=50 \%$ de $I, I_{2}=$ $100 \%$ de $I$ e $I_{3}=150 \%$ de $I$. Os níveis de nitrogênio foram $N_{0}=0, N_{1}=80, N_{2}=160$ e $N_{3}=240$ $\mathrm{kg} \cdot \mathrm{ha}^{-1}$. A aplicação de agua foi feita na forma de aspersão, utilizando um tubo de PVC perfurado, de $1 / 2$ " de diâmetro, em forma de $T$, com furos de $1 \mathrm{~mm}$ espaçados de $1 \mathrm{~cm}$, conforme descrito por TEODORO (1991).

$\mathrm{Na}$ fase de estabelecimento da cultura, foram realizadas irrigações uniformes em todos os tratamentos, com turnos de rega de dois dias, até as plantas atingirem a altura de $10 \mathrm{~cm}$ (20 dias após a semeadura), totalizando uma lâmina de água de $30 \mathrm{~mm}$. A partir dessa data as irrigações foram diferenciadas nos níveis estabelecidos.

Efetuaram-se dois cortes visando à produção de forragem; o primeiro com 50 dias apbs a semeadura e o segundo corte 40 dias após o primeiro. A altura de corte foi de $10 \mathrm{~cm}$ e a forragem produzida foi imediatamente pesada, para determinação da produção de matéria verde por subparcela. Do material verde retirou-se uma amostra de cada subparcela que, após pesagem, foi conduzida à estufa com ventilação forçada, para secagem à $70^{\circ} \mathrm{C}$, por 72 horas, para determinação da matéria seca.

Estudou-se a produtividade de matéria seca de aveia em função de dois fatores: água e nitrogênio. Pela análise de regressão ajustou-se o seguinte modelo:

$$
\begin{aligned}
& \mathbf{Y}(\mathbf{W}, \mathbf{N})=b_{o}+b_{1} \mathbf{W}+b_{2} \mathbf{N}+b_{11} \mathbf{W}^{2}+ \\
& +b_{22} \mathrm{~N}^{2}+b_{12} \mathbf{W N}
\end{aligned}
$$

em que:

$\mathbf{Y}(\mathbf{W}, \mathbf{N})$ - produtividade total de matéria seca de aveia, em t.ha' ${ }^{-1}$;

W - lâmina total de água, em mm; $e$

$\mathbf{N}$ - dose de nitrogênio, em kg.hat ${ }^{-1}$.

Considerando não haver restrições quanto à disponibilidade de mercado e de recursos para gastar com água e nitrogênio, determinaram-se as quantidades 6timas de de $\mathbf{W}$ e $N$, conhecidos $\mathbf{Y}(\mathbf{W}, \mathbf{N})$ e os preços do produto e dos insumos. Denominou-se quantidade ótima aquela que maximiza a receita líquida (ou margem de lucro), definida como a receita bruta da cultura menos os custos variáveis de aplicação da água e do nitrogênio. Para tal, formulou-se um modelo de programação linear separável, conforme McMILLAN Jr. (1970), considerando a função de produção e os seguintes parâmetros:

RL: receita líquida obtida com a cultura em $\mathbf{R} \$$. ha' $P_{0}$ : preço de 1 te forragem na lavoura, em R\$;

Cir custo de $1 \mathrm{~kg}$ de nitrogênio aplicado ao solo, em RS;

$\mathrm{C}_{\mathrm{m}}$ : custo de $1 \mathrm{~mm} \cdot \mathrm{ha}\left(10 \mathrm{~m}^{3}\right)$ de água aplicado ao solo, em R\$;

f : produtividade adicional ( $\left.\mathrm{kg} \cdot \mathrm{ha}^{-1}\right)$, devido apenas ab́ efeito da água, no passo $r$ da representação da porção separável da água;

f : produtividade adicional (kg.ha- $\left.{ }^{-1}\right)$, devido apenas ắ efeito do nitrogênio, no passo $s$ da representação da porção separável do nitrogênio;

f: produtividade adicional (kg.ha-1), devido apenas ẳ efeito da interação entre água e nitrogênio, no passo $t$ da representação da porção separável dessa interação;

$W_{r}$ : quantidade de água utilizada no passo $r$, em $\mathrm{mm}$; 
$\mathbf{N}$ : quantidade de nitrogênio utilizado no passo $s$, em kg.hat:

$\mathbf{V}$ : variável auxiliar que define a quantidade $\mathbf{V}$ utijlizada no passo $t$, sendo $V=W-N$;

$\lambda$ : variável que revela quanto do passo $r$ é adotado nąs representação da porção separável da água;

$\lambda$ : variável que revela quanto do passo s é adotado na representação da porção separável do njtrogênio; $e$

1 variável que revela quanto do passo té adotado na representação da porção separável de WN.

De acordo com informações obtidas junto a alguns produtores de aveia nos Estados de São Paulo e de Minas Gerais verificou-se que o preço recebido por 1 tonelada de forragem de aveia, nos meses janeiro a março de 1995, estava variando de R\$ 130,00 a R\$ 170,00 . Neste trabalho adotou-se um valor médio de $\mathbf{R} \$ 150,00$. Para o custo de 1 $\mathrm{kg}$ de nitrogênio aplicado ao solo, obteve-se junto a tais produtores um valor médio de $\mathbf{R} \$ \mathbf{0 , 5 0}$. Para o preço da água utilizou-se o valor médio de R\$ 0,20 , informado pelo INSTITUTO DE PESQUISAS TECNOLÓGICAS - IPT (1994) para áreas irrigadas por sistema pivô central no município de Guaíra (SP).

O problema consistiu em maximizar a seguinte função-objetivo:

$$
\mathbf{R} \mathbf{L}=\mathbf{P}_{o} \mathbf{Y}-\mathbf{C}_{n} \mathbf{N}-\mathbf{C}_{w} \mathbf{W}
$$

sujeito às restrições:

$\left.1 .^{\circ}\right)$ Se a interação WN não é significativa

$$
\begin{aligned}
& Y-\sum_{r=1}^{m} \lambda_{1 r} f_{1 r}-\sum_{s=1}^{m} \lambda_{2 s} f_{2 s} \leq b \\
& W-\sum_{r=1}^{m} \lambda_{1 r} W_{r} \geq 0 \\
& N-\sum_{s=1}^{\infty} \lambda_{2 s} N, \geq 0 \\
& \sum_{r=1}^{m} \lambda_{1 r} \leq 1 \mathrm{e} \lambda_{1 r} \geq 0
\end{aligned}
$$

$$
\sum_{s=1}^{n} \lambda_{2 s} \leq 1 \text { e } \lambda_{2 s} \geq 0
$$

$\left.2 .^{\circ}\right)$ Se a interação WN é significativa, a separabilidade da função é obtida fazendo-se:

$$
\mathbf{W N}=\frac{1}{2}\left[\mathbf{W}^{2}+\mathbf{N}^{2}-(\mathbf{W}-\mathbf{N})^{2}\right]
$$

A partir daí, define-se uma variável auxiliar $\mathbf{V}=$ $W \cdot N$. A função de produção terá três variáveis (W,N,V), envolvendo funções não lineares de todas elas. Aqui, acrescentam-se as seguintes restrições ao primeiro caso:

$$
\begin{aligned}
& v-\sum_{t=1}^{p} \lambda_{3 i} v_{t} \geq 0 \\
& \sum_{t=1}^{p} \lambda_{3 t} \leq 1 \text { e } \lambda \geq 0
\end{aligned}
$$

$\mathrm{Na}$ primeira restrição do problema sem interação, deve-se acrescentar ao primeiro membro o termo:

$$
-\sum_{t=1}^{p} \lambda_{3 t} f_{3 i}
$$

O modelo formulado utilizou grades de .pontos para $\mathrm{W}$ e $\mathbf{N}$ variando de 10 unidades a partir de zero, ate o limite de máxima produtividade.

\section{RESULTADOS E DISCUSSÃ̄o}

Os níveis de irrigação estabelecidos nas parcelas $(\mathfrak{\zeta})$ resultaram nas lâminas de água (IRR expressos na TABELA 1. Considerando os $30 \mathrm{~mm}$ de água aplicados durante o período de estabelecimento da cultura, as seguintes lâminas totais de irrigação (IRT I $_{1}$ foram utilizadas no período de produção de forragem (71dias): $I R T_{0}=30 \mathrm{~mm}$ para $I_{0} ; I_{R T}=144,5 \mathrm{~mm}$ para $I_{1}, I_{1 R T}=258,6$ $\mathrm{mm}$ para $\mathrm{I}_{2} \mathrm{e} / \mathrm{RT} \mathrm{T}_{3}=373,2 \mathrm{~mm}$ para $\mathrm{I}_{3}$. As análises foram realizadas considerando a precipitação pluvial acumulada (PREC) no período $(54,3 \mathrm{~mm}$ ), definindo-se, assim, as lâminas totais de água aplicadas (W = IRT + PREC). Em media, a freqüêencia de î́rigação (FREQ) foi de 9 dias. Nesse 
mesmo período, a evaporação acumulada no tanque "Classe A" foi de 282,9 mm.

Observa-se na TABELA 1 que o maior intervalo entre duas irrigações sucessivas (menor frequeência de irrigação) foi de 16 dias e ocorreu do $38 .{ }^{\circ}$ ao $54 .^{\circ}$ dia após o iń́cio dos tratamentos de irrigação. Condiçōes mais favoráveis ao consumo de água ocorreram entre o $10 .^{\circ} \mathrm{e} 16 .^{\circ}$ e entre $65 .^{\circ}$ e 71. ${ }^{\circ}$ dias após o início dos tratamentos de irrigação, com intervalo de 6 dias entre duas irrigações sucessivas. A precipitação pluviométrica acumulada durante o período experimental foi $19,19 \%$ da evaporação.

$\mathrm{Na}$ TABELA 2 estão apresentados os valores médios das produtividades de matéria seca para os dois cortes e produtividade total, em função das lâminas totais de água e dos níveis de nitrogênio. A análise de variância mostrou efeito significativo, ao nível de $1 \%$ de probabilidade, dos niveis de nitrogênio e das lâminas de água sobre a produtividade nos dois cortes e total. Não houve efeito significativo para a interação desses fatores.

Para o primeiro corte, a maior produtividade foi obtida no tratamento $\mathrm{W}_{3} \mathrm{~N}_{3}(3,455$ t.hat ${ }^{-1}$ e a menor em $W_{0} N_{0}\left(1,398\right.$ t.ha $\left.^{-1}\right)$. Para 0 segundo corte, a maior produtividade ocorreu no tratamento $W_{1} N_{1}\left(3,387\right.$ t. ha $\left.^{-1}\right)$ e a menor no tratamento $\mathrm{W}_{0} \mathrm{~N}_{0}\left(0,980\right.$ t.ha $\left.{ }^{-1}\right)$. Observa-se para os dois cortes que a ausência de irrigação prejudicou mais a produtividade de matéria seca, em relação a ausência da adubação nitrogenada.

As produtividades médias do primeiro corte foram superiores às do segundo, tanto para as doses de nitrogênio quanto para as lâminas de água, exceto na ausência de adubação nitrogenada que se teve produtividade maior no segundo corte. Estes resultados estão de acordo com os obtidos por VILELA et al. (1971), que conseguiram produtividades sensivelmente superiores para 0 primeiro corte em relação ao segundo. Entretanto, ANDRADE et al. (1975) conseguiram maiores produtividades no segundo corte. Comparando-se as produtividades entre os dois cortes, por tratamento, verifica-se que não foram mantidos os maiores valores para o primeiro corte em todos os tratamentos; em alguns as maiores produtividades ocorreram para o segundo corte.

Para a produtividade total, os tratamentos $W_{2} N_{2} \in W_{1} N_{1}$ resultaram nas maiores produtividades $\left(6,364\right.$ e 6,291 t.ha- $\left.{ }^{-1}\right)$, não diferindo estatisticamente entre si. As menores produtividades foram encontradas nos tratamentos $W_{6} N_{b}$ e $W_{0} N_{1}$ $\left(2,378\right.$ e 2,691 t.hat $\left.{ }^{-1}\right)$.

Definiu-se a função de produção de matéria seca de aveia utilizando-se o modelo polinomial quadrático. Na TABELA 3 mostram-se

TABELA 1. Lâminas totais de água aplicadas em cada parcela, frequêencia de irrigação e valores de algumas variáveis climáticas durante o período experimental.

\begin{tabular}{|c|c|c|c|c|c|c|c|c|}
\hline \multirow[t]{2}{*}{ DAT } & \multirow[t]{2}{*}{ DATAS } & \multirow{2}{*}{$\begin{array}{r}\text { PREC } \\
(\mathrm{mm})\end{array}$} & \multirow{2}{*}{$\begin{array}{r}\text { ECA } \\
(\mathrm{mm})\end{array}$} & \multicolumn{4}{|c|}{ LÂMINAS DE IRRIGAÇÃO (mm) } & \multirow{2}{*}{$\begin{array}{l}\text { FREQ } \\
\text { (dias) }\end{array}$} \\
\hline & & & & IRR $_{\mathbf{0}}$ & IRR $_{\text {I }}$ & IRR $_{2}$ & $\mathrm{IRR}_{3}$ & \\
\hline$\overline{0}$ & $30 / 04$ & 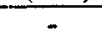 & - & - & - & - & - & \\
\hline 10 & $10 / 05$ & 28,5 & 59,3 & 0,0 & 15,4 & 30,8 & 46,2 & 10 \\
\hline 16 & $16 / 05$ & 0,0 & 28,0 & 0,0 & 14,0 & 28,0 & 42,0 & 6 \\
\hline 23 & $23 / 05$ & 0,0 & 27,9 & 0,0 & 14,0 & 27,9 & 42,0 & 7 \\
\hline 30 & $30 / 05$ & 0,0 & 27,2 & 0,0 & 13,6 & 27,2 & 40,8 & 7 \\
\hline 38 & $08 / 06$ & 6,1 & 33,2 & 0,0 & 13,6 & 27,1 & 40,7 & 8 \\
\hline 54 & $24 / 06$ & 16,2 & 46,2 & 0,0 & 15,0 & 30,0 & 45,0 & 16 \\
\hline 65 & $05 / 07$ & 3,5 & 31,0 & 0,0 & 13,8 & 27,5 & 41,3 & 11 \\
\hline 71 & $11 / 07$ & 0,0 & 30,1 & 0,0 & 15,1 & 30,1 & 45,2 & 6 \\
\hline \multicolumn{2}{|c|}{ TOTAL } & 54,3 & 282,9 & 0,0 & 114,5 & 228,6 & 343,2 & \\
\hline \multicolumn{4}{|c|}{$\mathbf{R T}_{1}=\sum \mathrm{IRR}_{1}+30,0$} & 30,0 & 144,3 & 258,6 & 373,2 & \\
\hline \multicolumn{4}{|c|}{$\mathrm{W}_{1}=\mathrm{IRT}_{1}+\mathrm{PREC}$} & 84,3 & 198,8 & 312,9 & 427,5 & \\
\hline
\end{tabular}

DAT - Dias após o início dos tratamentos de irrigação. FREQ - Frequiência de irrigação. IRR - Lâmina de irrigação correspondente ao nível $\mathrm{I}_{\mathrm{i}}$; IRT $\mathrm{I}_{1}$ - Lâmina total de irrigação. $\mathrm{W}_{1}$ - Lâmina total de água. PREC Precipitação pluvial acumulada. ECA - Evaporação acumulada no tanque "Classe $A$ ". 
as análises de variância da regressão $e$ de significância dos coeficientes das variáveis. A função encontrada foi:

$$
\begin{aligned}
& Y(W, N)=3,575 \cdot 10^{-2} W+1,554.10^{-2} N- \\
& -5,600.10^{-5} W^{2}-5,100.10^{-5} N^{2}
\end{aligned}
$$

sendo:

Y - produtividade de matéria seca, em t.ha ${ }^{-1}$;

W - lâmina total de água, em $\mathrm{mm}$; $e$

$\mathbf{N}$ - dose de nitrogênio, em kg.ha-1.

A produtividade máxima de matéria seca de aveia, estimada pela equação de regressão foi de 6,9 t.ha', obtida com a lâmina de água de $319,2 \mathrm{~mm}$ e a dose de nitrogénio de $152,4 \mathrm{~kg} \cdot \mathrm{ha}^{-1}$.
Tal valor de lâmina total de água corresponde a uma lâmina de irrigação de $264,9 \mathrm{~mm}$, aplicada desde a semeadura ou $234,9 \mathrm{~mm}$ após os 20 dias de estabelecimento da cultura, o que representou, em média, uma irrigação de $102,8 \%$ de ECA PREC, toda vez que esta diferença atingiu, aproximadamente, $30 \mathrm{~mm}$.

Na Figura 1 está representada a superfície de resposta num gráfico de duas dimensões, ao que se denomina curvas de isoproduto (ou isoquantas). As linhas de isoproduto mostram as diversas combinações de lâmina totais de água e doses de nitrogênio que resultaram numa mesma produtividade. A variação na declividade das isoquantas, ao longo de uma mesma linha reta, traçada à partir da origem, sugere que a combinação dos dois fatores, para a qual obtem-se menor custo,

TABELA 2. Produtividade de matéria seca de aveia (t.ha $\left.{ }^{-1}\right)$ em função das lâminas de água e dos níveis de

\begin{tabular}{|c|c|c|c|c|c|c|}
\hline \multirow{3}{*}{$\begin{array}{l}\text { (\% ECA) } \\
\text { (\% }\end{array}$} & \multirow{3}{*}{$\underset{(\mathrm{mm})}{W}$} & \multicolumn{4}{|c|}{ NIVEIS DE NITROGÊNIO (kg.ha-1) } & \multirow[t]{3}{*}{ MEDIAS } \\
\hline & & $\mathbf{0}$ & 80 & 160 & 240 & \\
\hline & & \multicolumn{4}{|c|}{$1 .^{\circ}$ CORTE } & \\
\hline $\begin{array}{l}0 \\
50 \\
100 \\
150 \\
\text { MÉDIAS }\end{array}$ & $\begin{array}{r}84,3 \\
198,6 \\
312,6 \\
427,2\end{array}$ & $\begin{array}{l}\text { B } 1,398 \mathrm{~b} \\
\text { B } 1,772 \mathrm{ab} \\
\text { C } 2,191 \mathrm{a} \\
\text { C } 2,367 \mathrm{a} \\
\text { B } 1,932\end{array}$ & $\begin{array}{l}\text { AB } 1,659 \text { b } \\
\text { A 2,904 a } \\
\text { B 2,901 a } \\
\text { B 3,067 a } \\
\text { AB 2,632 }\end{array}$ & $\begin{array}{l}\text { A } 1,894 \mathrm{~b} \\
\text { A } 3,212 \mathrm{a} \\
\text { A } 3,470 \mathrm{a} \\
\text { B } 3,062 \mathrm{a} \\
\text { A } 2,909\end{array}$ & $\begin{array}{l}\text { AB } 1,812 \text { b } \\
\text { A } 2,952 a \\
\text { AB } 3,209 a \\
\text { A } 3,455 a \\
\text { AB } 2,857\end{array}$ & $\begin{array}{l}1,690 \mathrm{~b} \\
2,710 \mathrm{a} \\
2,943 \mathrm{a} \\
2,987 \mathrm{a} \\
2,592\end{array}$ \\
\hline
\end{tabular}
nitrogênio.

2. ${ }^{\circ}$ CORTE

\begin{tabular}{lcllllll}
\hline 0 & 84,3 & B 2,980 b & AB 1,032 b & A 1,309 b & AB 1,265 b & $1,146 \mathrm{~b}$ \\
50 & 198,6 & BC 2,620 a & A 3,387 a & B 2,915 a & C 2,528 a & $2,852 \mathrm{a}$ \\
100 & 312,6 & B 2,586a & A 2,936 ab & AB 2,894 a & AB 2,870 a & $2,821 \mathrm{a}$ \\
150 & 427,2 & B 2,395 a & A 2,849 b & AB 2,568 a & B 2,451 a & $2,565 \mathrm{a}$ \\
MÉDIAS & & B 2,145 & A 2,551 & AB 2,421 & AB 2,278 & 2,348
\end{tabular}

PRODUTIVIDADE TOTAL

\begin{tabular}{lclllll}
\hline $\mathbf{0}$ & 84,3 & B 2,378 b & AB 2,691 b & A 3,203 b & AB 3,078 b & 2,837 b \\
50 & 198,6 & D 4,393 a & A 6,291 a & AB 6,127 a & C 5,480 a & 5,572 a \\
100 & 312,6 & C 4,777 a & AB 5,838 a & A 6,364 a & AB 6,081 a & 5,765 a \\
150 & 427,2 & C 4,762 a & A 5,917 a & AB 5,631 a & AB 5,906 a & 5,554 a \\
\hline MÉDIAS & & C 4,077 & AB 5,184 & A 5,331 & AB 5,136 & 4,932 \\
\hline \hline
\end{tabular}

Obs: (a) Considerando-se as médias na horizontal, os valores antecedidos da mesma letra maiúscula não diferem significativamente, ao nível de $5 \%$ de probabilidade, pelo teste de Tukey.

(b) Considerando-se as médias na vertical, os valores seguidos da mesma letra minúscula não diferem significativamente, ao nível de $5 \%$ de probabilidade, pelo teste de Tukey. 


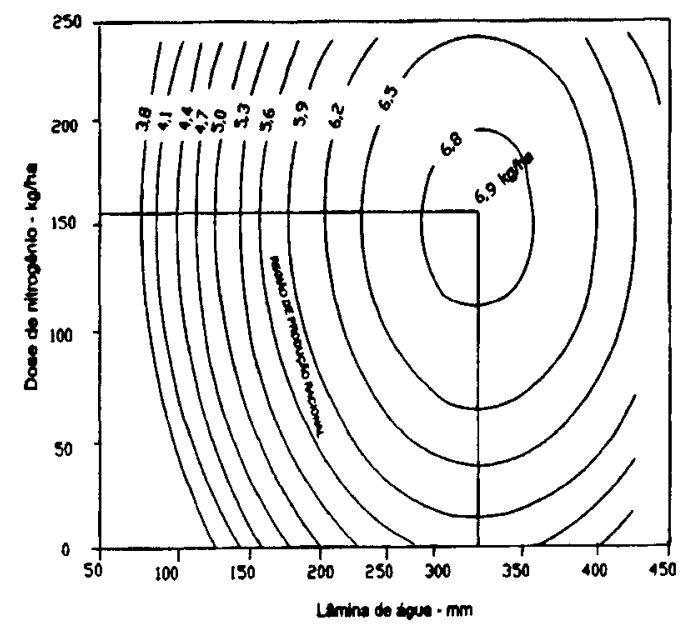

Figura 1 - Curvas de isoproduto e região de produção racional

varia com o nível de produtividade. Assim, a combinação ótima de lâmina de água e dose de nitrogênio para produzir 6,8 t.ha $^{-1}$ é diferente daquela para produzir 6,2 t.ha ${ }^{-1}$.

$\mathrm{Na}$ Figura 1 estão representadas as isóclinas que passam pelos pontos $\mathrm{dW} / \mathrm{dN}=0 \mathrm{e}$ $\mathrm{d} \mathbf{N} / \mathrm{d} \mathbf{W}=\mathbf{0}$ (taxas marginais de substituição de $\mathbf{W}$ por $\mathbf{N}$ são infinito e zero), definindo as duas fronteiras da região de produção racional. Dentro dessa região aplica-se o princípio de economizar; as taxas marginais de substituição de fatores são

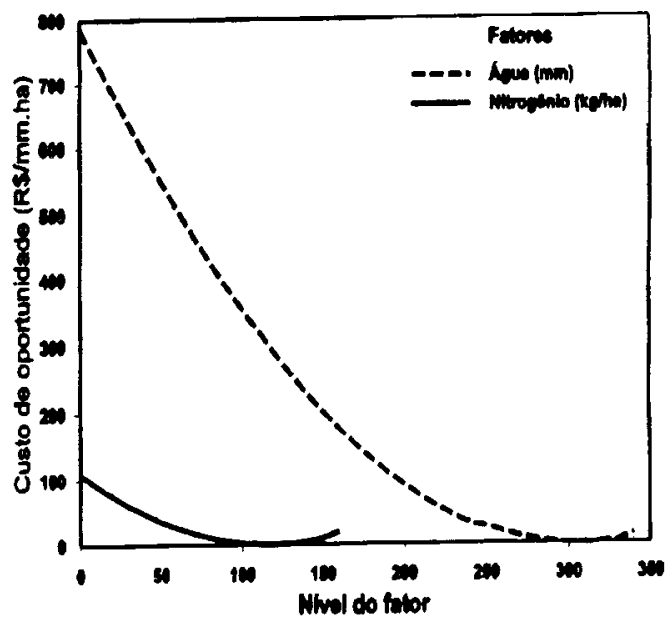

Figura 2 - Custos de oportunidades para água e nitrogênio

negativas o que implica dizer que $\mathbf{W}$ e $\mathbf{N}$ são substitutos. Ultrapassadas essas isóclina, a declividade da isoquanta é positiva, isto é, quantidades crescentes de ambos os fatores são empregados mantendo-se a produtividade no mesmo nível. Dispondo-se de uma importância fixa para gastar em água e nitrogênio, e podendo-se transferir gastos de um para outro fator, o custo mínimo para obter uma dada produtividade $Y$, é o custo correspondente à reta de isocusto que tangencia a isoquanta para $\mathbf{Y}$. A combinação dos fatores $\mathbf{W}$ e

TABELA 3. Análise de variância e coeficientes da regressão.

\begin{tabular}{lccc}
\hline \hline \multicolumn{4}{c}{ ESTIMATIVA DOS PARÂMETROS DA REGRESSÃO Y $=\mathrm{Y}(\mathrm{W}, \mathrm{N})$} \\
\hline COEFICIENTES & VALOR & ERRO PADRÃO & VALOR “ $*$ \\
\hline $\mathrm{B}_{0}$ & $-4,263 \times 10^{-1} \mathrm{~N} . S$ & $5,984 \times 10^{-1} * *$ & $-0,7124$ \\
$\mathrm{~B}_{1}$ & $3,575 \times 10^{-2} * *$ & $4,577 \times 10^{-3} * *$ & 7,8111 \\
$\mathrm{~B}_{2}$ & $1,554 \times 10^{-2} * *$ & $4,998 \times 10^{-3 * *}$ & 3,1083 \\
$\mathrm{~B}_{11}$ & $-5,600 \times 10^{-5 * *}$ & $8,485 \times 10^{-6 * *}$ & $-6,6440$ \\
$\mathrm{~B}_{22}$ & $-5,100 \times 10^{-5} * *$ & $1,700 \times 10^{-5 * *}$ & $-2,9351$ \\
$\mathrm{~B}_{12}$ & $3,217 \times 10^{-6} \mathrm{~N} . S$ & $9,701 \times 10^{-6 * *}$ & 0,3316 \\
& & &
\end{tabular}

ANÁLISE DA VARIÂNCIA DA REGRESSÃO $Y=Y(W, N)$

\begin{tabular}{lllll}
\hline CAUSA DA VARIAÇÃOS.Q. & G.L. & Q.M. & VALOR “F” \\
REGRESS ÃO & 26,5225 & 5 & 5,30451 & $26,9672 * *$ \\
ERRO & 1,96702 & 10 & 0,19670 &
\end{tabular}

$R^{2}=0.8964$

** - Indica significativo ao nível de $1 \%$ de probabilidade.

N.S - Indica não significativamente diferente de zero aos níveis usuais. 
TABELA 4. Intervalo 6́timo para os coeficientes da função-objetivo, em R\$.

\begin{tabular}{lccr}
\hline \hline VARIÁVEL & VALOR MÍNIMO & VALOR ORIGINAL & VALOR MÁXIMO \\
\hline Preço do produto (R\$/t) & 131,60 & 150,00 & 178,60 \\
Custo da água (R\$/mm.ha) & 0,08 & 0,20 & 0,24 \\
Custo do nitrogênio (R\$/kg) & 0,42 & 0,50 & 0,57 \\
\hline
\end{tabular}

$N$ correspondente ao ponto de tangência é a combinação de custo mínimo.

Não se dispondo de limitações de capital para gastar com $\mathbf{W}$ e $\mathbf{N}$, aos preços considerados, a otimização da função de produção resultou numa renda líquida máxima de $R \$$ $896,95 \mathrm{ha}^{-1}$. Este valor ocorreu quando foram aplicados $310 \mathrm{~mm}$ de água e $120 \mathrm{~kg} \cdot \mathrm{ha}^{-1} \mathrm{de}$ nitrogênio. $O$ uso desses insumos em tais níveis proporcionou uma produtividade de matéria seca de aveia de 6,8 t. ha ${ }^{-1}$. Foram também obtidas informações sobre o custo de oportunidade do uso de água e nitrogênio fora dos níveis ótimos (Figura 2). Por exemplo, quando não se utiliza água e nitrogênio ocorre uma redução de R\$ 787,45 mais R\$ $109,50$. ha $^{-1}$, respectivamente (R\$ 896,95), na função objetivo. Estes valores diminuem com a aplicação de agua e nitrogênio até o nível ótimo e começam a crescer novamente depois deste ponto. Utilizando-se $100 \mathrm{~mm}$ de água e $60 \mathrm{~kg} \cdot \mathrm{ha}^{-1}$ de nitrogênio a redução na função-objetivo será de $R$ \$ $384,35 \mathrm{ha}^{-1}$ (respectivamente, R\$ 335,20 e R\$ $\left.27,15 \mathrm{ha}^{-1}\right)$. Além do ponto 6́timo também obtémse redução na função-objetivo.

Informações sobre os intervalos de otimização para os coeficientes da função-objetivo constam na TABELA 4. Observa-se que preço do produto pode variar entre R\$ 131,60 e R\$ 178,60t-1 e a produtividade ainda permanece ótima. Da mesma forma, o preço da água pode variar entre $\mathrm{R} \$ 0,08 \mathrm{e} \mathbf{R} \$ 0,24$. mm.ha-1 $\mathrm{e}$ o preço do nitrogênio entre R\$ 0,42 e R\$ $0,57 \mathrm{~kg}^{-1}$ sem alterar a solução ótima.

\section{CONCLUSÕES}

a) A produtividade total de matéria seca de aveia seguiu um modelo quadrático, em relação às lâminas totais de água e dos níveis de nitrogênio, com valor máximo estimado de 6,9 t.ha- ${ }^{-1}$, para $319,2 \mathrm{~mm}$ de água e 152,4 kg.ha de nitrogênio. b) Os efeitos da irrigação na produtividade de matéria seca da aveia superaram os efeitos da adubação nitrogenada. $\mathrm{Na}$ ausência de irrigação, a adubação nitrogenada não promoveu aumento substancial na produtividade.

(c) Aos preços considerados para insumos e produto, a lâmina de água e a quantidade de nitrogênio que maximizaram a receita líquida ( $R \$$ $897,00 \mathrm{ha}^{-1}$ ) foram $310 \mathrm{~mm}$ e $120 \mathrm{~kg} \cdot \mathrm{ha}^{-1}$, respectivamente, correspondendo a produtividade ${ }^{1}$ de 6,8 tha ha $^{-1}$.

(d) Variando o preço do produto entre $R \$ 131,60 \mathrm{e}$ $\mathrm{R} \$ 178,60 . \mathrm{t}^{-1}$, o nível de produtividade de 6,8 t. $\mathrm{ha}^{-}$ ainda permaneceu ótimo. A solução b́tima não se alterou para os preços da água variando entre $R \$$ 0,08 e $R \$ 0,24 \mathrm{~mm}^{-1} \cdot \mathrm{ha}^{-1}$ e do nitrogênio entre $R \$$ 0,42 e $R \$ 0,57 \mathrm{~kg}^{-1}$.

\section{REFERÊNCIAS BIBLIOGRÁFICAS}

ALVIM, M. J.; GARDNER, A. L.; CÓSER, A. C. Estabelecimento e manejo de forrageiras de inverno sob pastejo. Coronel Pacheco, EMBRAPA, CNPGL, 1987. 22p.

ANDRADE, E. F.; PEREIRA, J. G.; CARVALHO, $M$. M.; LAMSTER, E. C. Competição entre forrageiras de inverno: Revista da Sociedade Brasileira de Zootecnia., v.4, n.1, p.1-11, 1975.

BLOUNT, G. G.; FISHER, M. J. Production and utilization of oats as forage for cattle in the Ord River Valley, Wester Australia. Australian Journal of Experimental Agriculture Animal Husbandry, v.16, n.78, p.88-93, 1976.

CROWDER, L. V.; LOTERO, J.; FRANSEN, J.; KRUK, C. F. Oats forage production in cool tropics as represented by Colombia. Agronomy Joumal, v. 59, n.1, p. 80-2, 1967.

CRUCIANI, D. E. \& MINAMI, K. Efeitos do excesso de água no solo na produção de aveia. Item, n.16, p.31-3, 1984. 
FLOSS, E. L. Aveia. In: BAIER, E. L.; FLOSS, E. L.; AUDE, M. I. S. As lavouras de inverno. Rio de Janeiro: Ed. Globo, 1988. p.16-74.

GILL, P. S.; MALIK, B. S. Response of oat varieties to soil moisture regimes and nitrogen levels. Forage Research, v.9, n.2, p.151-4, 1983.

GODOY, R.; BATISTA, L. A. R. Recomendação de cultivar de aveia forrageira para a região de São Carlos. In: REUNIĀO ANUAL DA SOCIEDADE BRASILEIRA DE ZOOTECNIA, 26., Porto Alegre, 1989. Anais. Porto Alegre, SBZ, 1989. p.35.

HUKKERI, S. B.; SHUKLA, N. P.; RAPUT, R. K. Effects of levels of soil moisture and nitrogen on the fodder yield of oat on two types of soil. Indian Journal of Agricultural Science, v.47, n.4, p.204-9, 1977.

INSTITUTO DE PESQUISAS TECNOLÓGICAS DO ESTADO DE SÃO PAULO. Racionalizaçăo do uso da água de irrigaçăo nos municípios de Guaíra e Casa Branca, SP, São Paulo, v.2, 27p., 1994. (IPT, Relatório Técnico, 30254).

LAL, M. Studies on irrigation scheduling in mited stands of forage oat and legumes. Indian Agronomy Journal., v.32, n.1, p.21-3, 1987.

LEITE, N. Culturas forrageiras não convencionais e sua utilização. In: SIMPÓsIO SOBRE MANEJO DA PAStageM, 8., Piracicaba, 1986. Resumos. Piracicaba: FEALQ, 1986. p.49-75.
McMILLAN Jr, C. Mathematical programming. New York: John Wiley, 1970. 496p.

NAsCIMENTO, C. M.; PEREIRA, M. A. M. G. Atlas climático do Estado de Săo Paulo. Campinas: Fundação Cargill, 1988. 93p.

OLIVEIRA, J. B.; PRADO, H. Levantamento pedológico do Estado de São Paulo: Quadrícula de São Carlos. II Memorial descritivo. Campinas: IAC, 1984. 188p (IAC. Boletim Técnico, 98)

TEODORO, R. E. F. Irrigação e adubaçāo nitrogenada na produção de forragem e grũos de aveia (Avena sativa L.). Botucatu, 1991. 137p. Tese (Doutorado) Faculdade de Ciências Agronômicas, Universidade Estadual Paulista "Júlio de Mesquita Filho".

VILELA, H.; MOREIRA, H. A.; VELOSO, J. A. F. PEREIRA, C. S. Aveia (Avena sativa L.) como forrageira de inverno: Efeito da adubação e irrigação em solo de cerrado. Arquivos da Escola de Veterinária da Universidade Federal de Minas Gernis, v.22, p.103-11, 1970.

VILELA, H.; BARBOSA, A. S.; CAVALCANTE, S. S.; ANDRADE, V. J.; VELOSO, J. A. F.Aveia (Avena sativa, 1.) como forrageira de inverno: Efeito de três épocas de plantio sobre a produção. Arquivos da Escola de Veterinária da Universidade Federal de Minas Gerais., v.23, p.59-66, 1971.

Recebido para publicação em 23.06.95

Aceito para publicação em 27.02.95 